\title{
False-positive uptake of radioiodine WBS in a patient with papillary thyroid cancer due to a vertebral hemangioma
}

\author{
E. Triantafillou(1), F. Kanouta(1), G. Papadakis ${ }^{(1)}$,I. Tzaves(1), E. Panagiotidi(1), E. Trivizaki( ${ }^{(2)}$, \\ V. Kaltzidou(1), A. Tertipi ${ }^{(1)}$, A. Drosou ${ }^{(1)}$, A. Pappas ${ }^{(1)}$
}

Endocrinology Department ${ }^{(1)}$ and Department of Nuclear Medicine ${ }^{(2)}$, Metaxa Anticancer Hospital, Pireaus, Athens, Greece

Introduction:

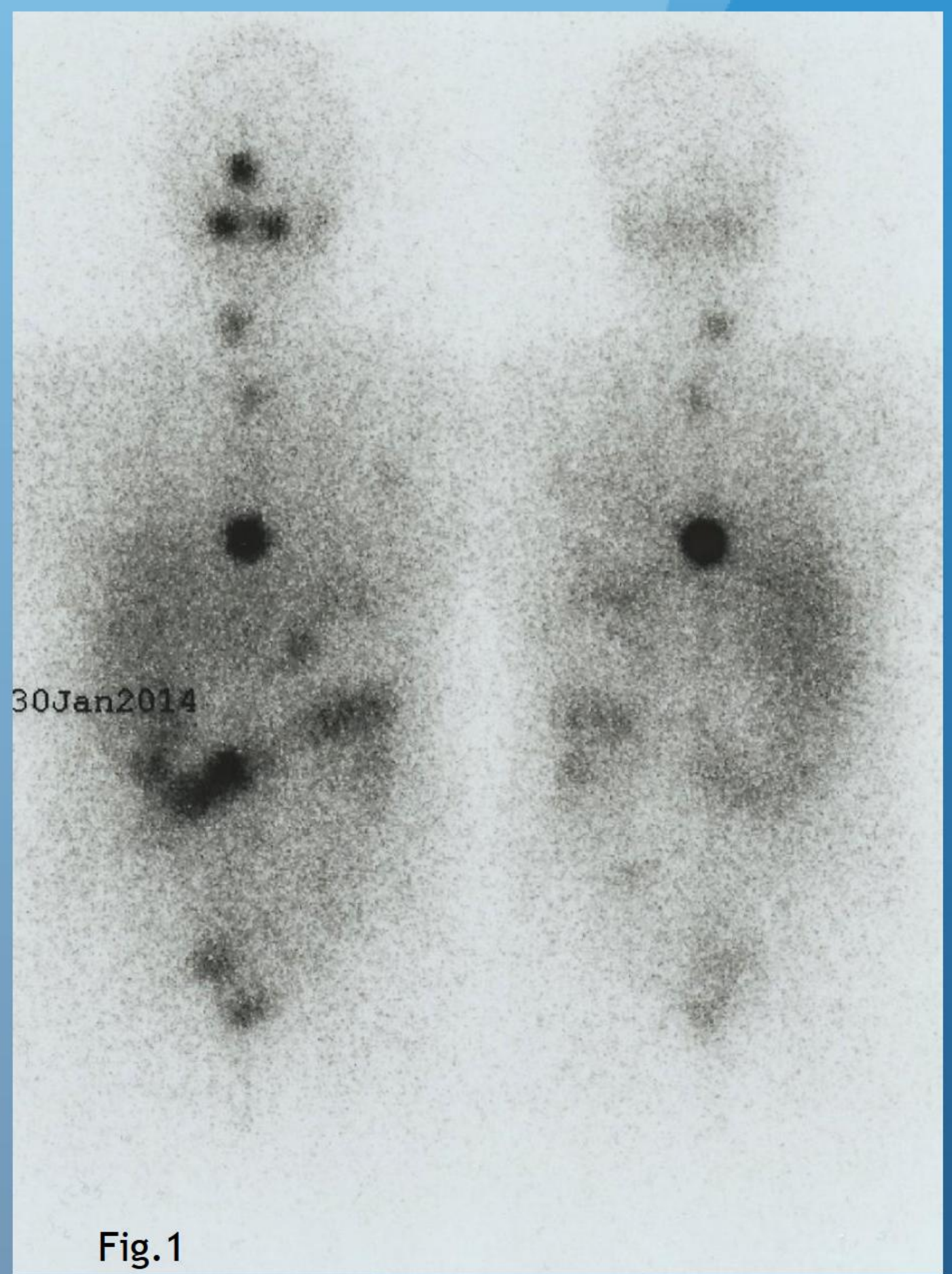

Fig. 1

\section{References}

1)Carballo M, Quiros RM 2012 To Treat or Not to Treat: The Role of Adjuvant Radioiodine Therapy in Thyroid Cancer Patients J Oncol

2) Verbeke SL, Bovée JV. 2011 Primary vascular tumors of bone: a spectrum of entities? Int J Clin Exp Pathol 4:541-551

3) Vinay S, Khan SK, Braybrooke JR 2011 Lumbar vertebral haemangioma causing pathological fracture, epidural haemorrhage, and cord compression: a case report and review of literature J Spinal Cord Med. 34:335-339

4) Laguna R, Silva $F$ et al 2000 Vertebral hemangioma mimicking a metastatic bone lesion in well-differentiated thyroid carcinoma. Clin Nucl Med;25(8):611-613.

5) Giuffrida D et al 1993 False positive 131 total body scan due to an ectasia of the common carotidis. J Endocrinol Invest 16:207-11
Diagnostic and post-therapy radioiodine whole body scan (WBS) has traditionally been used as an integral diagnostic procedure in the follow-up of differentiated thyroid carcinoma (DTC) for the detection of local recurrence or remote matastasis (1). Regularly therapeutic

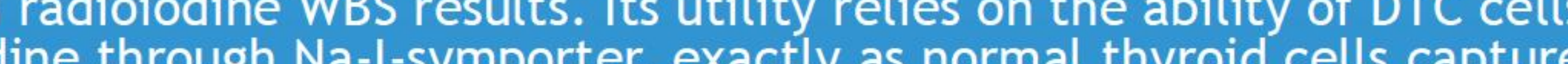
iodine from the blood. Other tissues such as the salivary glands, nasopharynx, gastric mucosa or the mammary gland posses the same symporter. Hence, a rather faint visualization of these organs on radioiodine WBS is considered normal. However, there are several reports in the literature of false positive uptake of radioiodine WBS in patients with DTC due to physiologic or pathologic conditions unrelated to DTC. We report a case of a 37-year old female patient with papillary thyroid cancer who presented with an abnormal radioiodine uptake on eighth thoracic vertebrae due to an atypical vertebral

Case Report:

A 37-year-old female patient underwent subtotal thyroidectomy for multinodular goiter A histology revealed multifocal papillary thyroid carcinoma, of follicular type with three foci of major diameter $1.35 \mathrm{~mm}$. Post-operative radioiodine uptake of 24 hours was $2.7 \%$ and thyroglobulin $(\mathrm{Tg})$ levels were $35 \mathrm{ng} / \mathrm{ml}$. Neck ultrasound was negative for pathologic ymph nodes and chest X-ray was unremarkable. An ablation dose of $70 \mathrm{mCi}$ radioiodine was

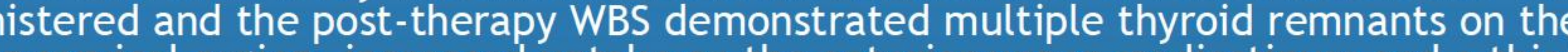

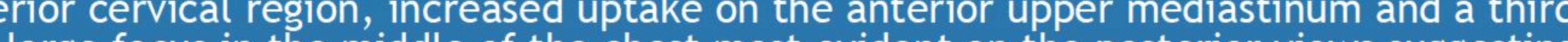
vertebral metastatic involvement (Figure 1). Chest CT scan, cervical and mediastinal MR were negative. In the 99Tc-bone scan a small focus of increased uptake was observed at the $8^{\text {th }}$ thoracic vertebrae with no other pathologic findings of the skeleton. MRI of the spine showed a high intensity pathologic signal in the $8^{\text {th }}$ thoracic vertebrae with a major

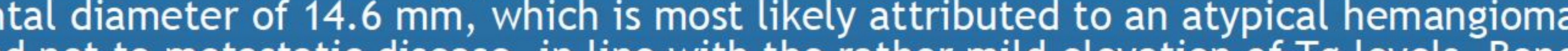
and not to metastatic disease, in line with the rather mild elevation of Tg levels. Bone metastases in DTC are known to cause higher Tg concentrations, usually $>100 \mathrm{ng} / \mathrm{ml}$.

Discussion:

Vertebral hemangiomas are common and autopsy studies report a prevalence of approximately $10 \%$ in the general population, with females affected more often (2). istopathologically, vertebral hemangiomas are benign tumors with a predilection for the vertebra in our patient was due to a vertebral hemangioma and not to metastatic disease, in consent with the rather mild elevation of Tg levels. Bone metastasis in DTC are known to cause higher Tg concentrations, usually $>100 \mathrm{ng} / \mathrm{ml}$. Radioiodine uptake by a vertebral hemangioma in a patient with DTC has been reported in the literature in very few cases $(3,4)$. Assumptions regarding the functional-pathological mechanisms suggest pooling of the iodine-laden blood in the hemangioma vessels, an obvious mode, since other vesse with increased capillary permeability leading to transcapillary escape of iodine and interstitial retention. Finally, hemangioma vessels may have abnormal structure, facilitating exudation of radioiodine in the interstitial space. These false positive results may mislead the physician to the presence of distant metastasis and erroneously change the stage of the disease and the therapeutic approach. 